\title{
Sum-Throughput Maximization in Downlink OFDMA-based Cellular Cognitive Radio Networks
}

\author{
Fayezeh Ghavimi, IEEE Student Member
}

\begin{abstract}
We consider a downlink OFDMA-based cellular cognitive radio network $(\mathrm{CRN})$ coexisted with primary radio networks (PRNs). The goal is joint scheduling and power allocation among CRN users to maximize the sum-throughput of the CRN satisfying the interference constraints on the PRN. To this aim, first we propose cognitive single cell multiple access channel (CSCMAC) algorithm to find search area faster, where the goal is to find a simple, fast and stable method to calculate optimal dual variables in resource allocation for each individual user. Then, utilizing these values, we propose cognitive two cell iterative spectrum balancing (CTC-ISB) algorithm, where by considering interfering effects caused by adjacent cells as well as interference constraints on the primary network, the power and scheduling are allocated among the CRN users so that the sum-throughput of the CRN network is maximized. The proposed algorithm achieves near-optimal performance in practical applications. The proposed method is compared with other algorithms and simulation results are provided to assess its performance. Numerical results show that the proposed algorithm outperforms traditional algorithm in terms of sumthroughput maximization of the total network.
\end{abstract}

Keywords-Resource allocation, cellular cognitive radio network, orthogonal frequency division multiple access (OFDMA), non-convex optimization

\section{INTRODUCTION}

Traditional spectrum regulation assigns users to prescribed frequency bands, thus limiting the potential users to dynamically access allocated radio spectrum. This policy, together with the rapid deployment of various wireless services, leads to increasing scarcity in the radio spectrum. On the other hand, many available spectrum resources are not efficiently used [1]. The cognitive radio (CR) technique has been proposed as a promising solution [2], [3], that enables unlicensed users to access these spectrums temporarily and thus to improve overall spectrum efficiency under acceptable interference to the licensed users [2]-[7]. However, such spectrum access by secondary users (SUs) needs to avoid detrimental interference to the primary users (PUs). To flexibly implement spectral sharing between Pus and SUs and maximize the overall throughput of SUs, a dynamic spectrum allocation (DSA) design is required. Orthogonal frequency division multiple access (OFDMA) is a strong candidate for an efficient design in cellular cognitive radio networks. Various DSA methods were proposed for the optimization of CRN associated with primary radio network (PRN) [8]-[14].

Fayezeh Ghavimi is with the Engineering Science Department, National Cheng Kung University, Tainan, Taiwan (corresponding author's phone: +886983627120; e-mail: faiezeh.ghavimi@gmail.com).
Many past results on OFDMA-based DSA methods assumed the single cell scheme for CRNs [10]-[14]. In [11], several uplink scheduling algorithms which can reduce the SU-to-PU interference were proposed. In [13], a single cell collocated PRN/CRN downlink model was proposed and the weighted sum-rate (WSR) maximization of all PUs and SUs was studied using proportional fair scheduling (PFS) and sequential quadratic programming algorithm. In [14], both centralized and distributed algorithms were designed and the WSR for several SU links was optimized using the Lagrange duality optimization.

A heuristic interference minimization scheme with an innovative channel allocation method is proposed in [15] while minimizing the overall interference to the PUs. The authors in [16] investigated a joint power allocation and channel assignment scheme utilizing the heuristic method in [15] to minimize the aggregate interference from multiple femtocells to PUs while satisfying the constraints on throughput of each femtocell.

Outage probability has been widely studied for different CR scenarios [17]-[20]. For instance, the authors in [19], studied the outage probability performance of a multi-hop decode-and-forward (DF) spectrum sharing network with multiple antenna at the SUs. The authors in [21], studied the outage performance, bit error rate, and capacity performance for a multi-hop spectrum sharing network. The power constraint on the secondary transmit nodes as well as primary network interference constraint were ignored. The authors in [22] investigated the asymptotic outage and error performance in a dual-hop single antenna CR network. In this work, the effect of PUs and co-channel interferences were considered, but the effect of additive white Gaussian noise (AWGN) was ignored.

Most of the previous contributions focused on the outage performance only, and other performance metrics such as the ergodic capacity has not been studied thoroughly. Furthermore, despite the above contributions, the CRN optimization taking into account inter-cell interference management and the power spectral mask (PSM) constraints for multi-cell system has not been well studied. In multi-cell case, the inter-cell interference makes the problem more complex and solution of the problem cannot be obtained by ordinary methods. Furthermore, due to the inter-cell interference, the throughput of each cell not only depends on the power and subcarrier allocation of its own, but also depends on resource allocation in adjacent cells. In this type of problem, the resources should be managed to achieve the considering objective function and it needs to avoid causing detrimental interference to the PUs. Furthermore, respect to the users of adjacent cells, it is necessary to manage multi-cell interference. This type of optimization has a nonconvex structure and computing its globally solution may not be feasible in practice. The objective of this paper is to propose 
iterative algorithms to efficiently obtain near-optimal solution. Thus, in this paper, we propose low-complexity algorithms that use simplified and practical assumptions to make the problem easier. The following two iterative suboptimal algorithms are proposed to solve the problem.

1) Cognitive Single Cell Multiple Access Channel (CSCMAC): To find the search area faster, the CSC-MAC algorithm was proposed. In this method, it is assumed that subcarrier allocation is performed for each user and the power allocation is done and primary values for the dual variables in each cell are obtained. By obtaining these amounts, the search interval becomes more limited and as a result, the algorithm will converge to optimal values faster.

2) Cognitive two cells iterative spectrum balancing (CTCISB): By obtaining the search area, we proposed a second algorithm in which the purpose is to maximizing the network sum-throughput using the Lagrange dual optimization method. In this method, water-filling algorithm is performed for users in each cell according to the effects caused by the users in adjacent cell, and power allocation and subcarrier scheduling are performed. Then, the dual variables are updated using ellipsoid method is done and this process is repeated until the algorithm converges to optimal values. Such an implementation is quite attractive in practical systems since it reduces computational complexity. To compare the performance with other algorithms, we provide the performance of a single cell power allocation algorithm, which by considering interference limitation on primary network and disregarding interference caused by the users in adjacent cell, the sub-carrier is assigned to the user who has the better signal-to-interference ratio (SIR) on the considered sub-carrier. Also, we study the performance of equal power allocation algorithm, which by regarding interference constraint on primary network, power is allocated equally among users in each sub-carrier and also sub-carrier is allocated to the user that has better SIR. Simulation results show that the proposed algorithms have a higher capacity than the single cell power allocation and equal power allocation algorithm and also these algorithms provide large performance advantages than the two previous schemes. Moreover, the proposed duality schemes enjoy fast convergence and low complexity.

The remainder of this paper is organized as follows. Section II describes the system model. In Section III, the CSC-MAC and CTC-ISB algorithms are presented. Section IV, provides numerical results. Concluding remarks are given in Section V.

\section{SYSTEM MODEL}

Consider the system model in Fig. 1. Each PRN cell has one BS and multiple PUs, while each CRN cell has one BS and multiple SUs collocated with a PRN cell. Users and base stations are equipped with one receive and one transmit antenna, respectively. Each user is connected to only one base station that is selected based on long-term channel state information (CSI).

We denote the nonempty set of SU indices in cell $m$ by $B_{m}$ indicating $\mathrm{k} \in \mathrm{B}_{\mathrm{m}}$ when SU $k$ is located in CRN cell $m$. Let us consider a cluster of $M=2$ coordinated base station in an OFDMA cellular CRN that employs $N$ subcarriers. In each
CRN cell, the $\mathrm{k}_{\mathrm{m}}$ SUs communicate with BS $m$, and compete for a set of $\mathrm{N}_{\mathrm{m}}$ available frequency bands.

We consider a downlink OFDMA-based cellular cognitive radio network. In this case, users belonging to different sectors of the same cell are spatially orthogonal. Therefore, it is reasonable to assume that a user is only subject to interference from the nearest interfering base stations of CRN and PRN networks. In order to simplify, we focus on two cells, say cell $A$ and $B$ as shown in Fig.1. We denote the number of users in cell $A$ and $B$ by $\mathrm{K}^{\mathrm{A}}$ and $\mathrm{K}^{\mathrm{B}}$ respectively. The radius of each cell is denoted by $D$. For each user $\mathrm{k} \in\left\{1,2, \ldots, \mathrm{K}^{\mathrm{A}}\right\}$ of cell $A$, the signal received by $k$ at the $n$th subcarrier and at the $m$ th OFDM block is given by

$$
\begin{aligned}
& r_{k, n}^{A}[m]=H_{k, n}^{A}[m] \times x_{k, n}^{A}[m]+\bar{H}_{k, n}^{A}[m] \times \sum_{l=k_{n}^{B}} x_{l, n}^{B}[m] \\
& +z_{k, n}^{A}[m]
\end{aligned}
$$

where $H_{k, n}^{A}[m]=L_{k}^{A} \times g_{k, n}^{A}$ is the complex channel response between user $k$ in cell $A$ and base station $A$ at the subcarrier $n$ and the OFDM block $m$ including pass loss component $\mathrm{L}_{\mathrm{k}}^{\mathrm{A}}$ and Rayleigh fading $g_{k, n}^{A}$. The $K_{n}^{B}$ denotes the user assigned to subcarrier $n$ in cell $B$. The $\mathrm{x}_{\mathrm{k}, \mathrm{n}}^{\mathrm{A}}(\mathrm{m})$ is the complex symbol transmitted by base station $A$ to user $k$ on the subcarrier $n$. $\bar{H}_{k, n}^{A}[m] \times \sum_{l=K_{n}^{B}} x_{l, n}^{B}[m]$ represent inter-cell interference which coefficient $\overline{\mathrm{H}}_{\mathrm{k}, \mathrm{n}}^{\mathrm{A}}[\mathrm{m}]$ is channel response at the subcarrier $n$ and the OFDM block $m$ between user $k$ in cell $A$ and base station $B$ and $\mathrm{x}_{1, \mathrm{n}}^{\mathrm{B}}[\mathrm{m}]$ is the complex symbol transmitted by base station $B$ to user $k$ on the subcarrier $n$. Finally $\mathrm{z}_{\mathrm{k}, \mathrm{n}}^{\mathrm{A}}[\mathrm{m}]$ is an additive white Gaussian noise with zero mean and variance $\delta^{2}=\mathrm{N}_{0} \frac{\mathrm{B}}{\mathrm{N}}$ which $\mathrm{N}_{0}$ is power spectral density of thermal noise and $B$ is total bandwidth of the system.

Assume that each subcarrier is assigned to one user in each cell, thus the link capacity of user $k$ in subcarrier $n$ is

$$
C_{k, n}^{A}\left(P_{k, n}^{A}, P_{l, n}^{B}\right)=\log _{2}\left(1+\frac{g_{k, n}^{A} \cdot p_{k, n}^{A}}{N_{0}+I_{P}+\bar{g}_{k, n}^{A} p_{l, n}^{B}}\right)
$$

which $\mathrm{g}_{\mathrm{k}, \mathrm{n}}^{\mathrm{A}}$ and $\mathrm{p}_{\mathrm{k}, \mathrm{n}}^{\mathrm{A}}$ demonstrate the gain and power of user in subcarrier $n$ in cell $A$ respectively. The $\overline{\mathrm{g}}_{\mathrm{k}, \mathrm{n}}^{\mathrm{A}}$ and $\mathrm{p}_{1, \mathrm{n}}^{\mathrm{B}}$ indicate the gain and power of user in subcarrier $n$ in cell $B$ respectively. $I_{P}$ is interference caused by base stations of primary networks.

Hence, sum-throughput maximization problem in two adjacent cells is defined as follows. $\mathrm{P}_{\mathrm{T}}^{\mathrm{A}}$ and $\mathrm{P}_{\mathrm{T}}^{\mathrm{B}}$ are the total power constraint at base station $A$ and $B$ respectively. The first and second constraints indicate that sum power of users in all subcarriers should not exceed than the total power of base station in each cell. 


$$
\begin{gathered}
C\left(P_{k, n}^{A}, P_{l, n}^{B}\right)=\max _{p_{k, n}^{A}, p_{l, n}^{B}} \sum_{k=1}^{K_{k}^{A}, N_{l}^{B}} \sum_{n \in N_{k}^{A}} C_{k, n}^{A}+\sum_{l=1}^{K^{B}} \sum_{n \in N_{l}^{B}} C_{l, n}^{B} \\
\text { subject to }: \sum_{k=1}^{K^{A}} \sum_{n \in N_{k}^{A}} p_{k, n}^{A} \leq P_{T}^{A}, \quad \sum_{l=1}^{K^{B}} \sum_{n \in N_{l}^{B}} p_{l, n}^{B} \leq P_{T}^{B} \\
p_{k, n}^{A}, p_{l, n}^{B} \geq 0 \text { for all } k, n, l \\
p_{k, n}^{A} \leq \bar{I}_{n}^{A} \forall k, p_{l, n}^{B} \leq \bar{I}_{n}^{B} \forall l
\end{gathered}
$$

The third and fourth ensure nonnegative powers in subcarriers in each cell and constraints 5 and 6 ensure that power control is performed in each cell such that interference does not exceed a certain threshold in PUs system. Therefore, in this way, detrimental interference to the PUs could be avoided.

Due to that the problem (3) is a nonconvex combinatorial optimization, computing its globally optimal solution may not be feasible in practice. Thus, the objective of this paper is to propose iterative algorithms to efficiently obtain near-optimal solutions to the aforementioned problem (3).

\section{TWO-CELLS RESOURCE ALLOCATION}

In this section, we present two iterative strategies for joint scheduling and power allocation given that the complete CSI is available. The first algorithm which is referred to cognitive single cell multiple access channel (CSC-MAC) is monotonic and provably converges. The second algorithm which is based on dual decomposition can yield near-optimal value of (3) and in fact, the optimal value is obtained when $\mathrm{N} \rightarrow \infty$.

\section{A. Cognitive Single Cell Multiple Access Channel}

In this section, the aim is to allocate power assuming that power allocation does not depend on the power allocation in adjacent cell while satisfying the associated constraints in each cell. Thus, to maximize the sum-throughput of two cells problem (4) is constituted as follows.

$$
\begin{aligned}
& \max _{p_{k, n}^{A}, p_{l, n}^{B}}\left[\sum_{k=1}^{K^{A}} \sum_{n \in N_{k}^{A}} C_{k, n}^{A}\left(p_{k, n}^{A}\right)+\sum_{l=1}^{K^{B}} \sum_{n \in N_{l}^{B}} C_{l, n}^{B}\left(p_{l, n}^{B}\right)\right] \\
& \text { subject to }: \sum_{k=1}^{K^{A}} \sum_{n \in N_{k}^{A}} p_{k, n}^{A} \leq P_{T}^{A}, \sum_{l=1}^{K^{B}} \sum_{n \in N_{l}^{B}} p_{l, n}^{B} \leq P_{T}^{B} \\
& p_{k, n}^{A}, p_{l, n}^{B} \geq 0 \forall k, l, n \\
& p_{k, n}^{A} \leq \bar{I}_{n}^{A}, p_{l, n}^{B} \leq \bar{I}_{n}^{B} \forall k, l, n \\
& C_{k, n}^{A}\left(p_{k, n}^{A}\right)=\log _{2}\left(1+\frac{g_{k, n}^{A} \cdot p_{k, n}^{A}}{N_{0}+I_{P}}\right) \\
& C_{l, n}^{B}\left(P_{l, n}^{B}\right)=\log _{2}\left(1+\frac{g_{l, n}^{B} \cdot p_{l, n}^{B}}{N_{0}+I_{P}}\right) .
\end{aligned}
$$

Using standard optimization techniques in [23], we obtain the Lagrange function and by differentiating it regarding to $\mathrm{p}_{\mathrm{k}, \mathrm{n}}^{\mathrm{A}}$ and $\mathrm{p}_{1, \mathrm{n}}^{\mathrm{B}}$ the following results are obtained.

$$
\begin{aligned}
& p_{k, n}^{A^{*}}= \begin{cases}\bar{I}_{n}^{A}, & \sum_{n=1}^{N} \bar{I}_{n}^{A}<P_{T}^{A} \\
\min \left\{\left[\frac{1}{L n(2) \lambda_{\text {sin gle }}^{A}}-\frac{N_{0}+I_{p}}{g_{k, n}^{A}}\right]^{+}, \bar{I}_{n}^{A}\right\}, & \sum_{n=1}^{N} \bar{I}_{n}^{A} \geq P_{T}^{A}\end{cases} \\
& p_{l, n}^{B^{*}}= \begin{cases}\bar{I}_{n}^{B}, & \sum_{n=1}^{N} \bar{I}_{n}^{B}<P_{T}^{B} \\
\min \left\{\left[\frac{1}{\operatorname{Ln}(2) \lambda_{\text {sin gle }}^{B}}-\frac{N_{0}+I_{p}}{g_{k, n}^{B}}\right]^{+}, \bar{I}_{n}^{B}\right\}, \sum_{n=1}^{N} \bar{I}_{n}^{B} \geq P_{T}^{B}\end{cases}
\end{aligned}
$$

The optimal values of $\lambda_{\text {single }}^{\mathrm{A}}$ and $\lambda_{\text {single }}^{\mathrm{B}}$ are chosen to fulfill the total power constraint in each cell. By considering the power allocation formula in (5) and (6), we perform water filling algorithm in each cell. Then, we can use an optimization algorithm based on bisection method to obtain the optimal value of $\lambda_{\text {single }}^{\mathrm{A}}$ and $\lambda_{\text {single }}^{\mathrm{B}}$. In fact, we will use the optimal value of $\lambda_{\text {single }}^{\mathrm{A}}$ and $\lambda_{\text {single }}^{\mathrm{B}}$ to restrict the search area and decrease the time to obtain the optimal values for power allocation. The algorithm ensures that this values will converge to the optimal values. As a result, fast and stable convergence is achieved. The optimal power allocation algorithm in cell $A$ is derived in the following. The power allocation in cell $B$ is conducted in the same manner.

After that, the scheduling is performed which in order to maximize throughput of a single cell for any given feasible power allocation, the solution to

$$
\begin{gathered}
\max _{k_{n}^{A}} C_{k, n}^{A}\left(p_{k, n}^{A}\right) \\
\max _{l_{n}^{B}} C_{l, n}^{B}\left(p_{l, n}^{B}\right)
\end{gathered}
$$

is achieved at

$$
\begin{aligned}
& k_{n}^{A}=\underset{k \in K^{A}}{\arg \max }\left\{\log _{2}\left(1+\frac{g_{k, n}^{A} p_{k, n}^{A}}{N_{0}+I_{P}}\right)-\lambda^{A} p_{k, n}^{A}\right\} \\
& l_{n}^{B}=\underset{l \in K^{B}}{\arg \max }\left\{\log _{2}\left(1+\frac{g_{l, n}^{B} p_{l, n}^{B}}{N_{0}+I_{P}}\right)-\lambda^{B} p_{l, n}^{B}\right\}
\end{aligned}
$$

Algorithm 1: Associated Bisection Search Cognitive Single Cell Multiple Access Channel (CSC-MAC) Power Allocation

1: Initialize $k \in\{1,2, \ldots, K\}, n \in\{1,2, \ldots, N\}, \lambda_{\text {min }}=0$,

$\lambda_{\max }=\delta N, \delta>>1$

2: repeat

3: Update $\lambda_{\text {sin } g l e}^{A}=\frac{\lambda_{\min }+\lambda_{\max }}{2}$;

4: repeat

5: for each sub-carrier $\mathrm{n}=1$ to $N$ do

6: Calculate $P_{k, n}^{A^{*}}$ via (5);

7: $\quad$ Set $k_{n}^{A}$ and $P_{k, n}^{A}$ according to (9) and (5) respectively;

8: $\quad$ For $k_{n}^{A}=k_{n}^{A^{*}}$, set $P_{k, n}^{A}=P_{k, n}^{A^{*}}$ and $k_{n}^{A}=1$;

9: end for 
10: Update $\lambda_{\min }=\lambda_{\text {sin } g l e}^{A}$ if $\sum_{k=1}^{K^{A}} \sum_{n=1}^{N_{K}^{A}} P_{k, n}^{A}>P_{T}^{A}$, else $\lambda_{\max }=\lambda_{\sin g l e}^{A}$

11: Until required accuracy $\left|\sum_{k=1}^{K^{A}} \sum_{n=1}^{N_{k}^{A}} P_{k, n}^{A}-P_{T}^{A}\right|<\varepsilon$

In Algorithm $1, \varepsilon>0$ defines the error tolerance in the computed optimal water level. The algorithm converges if the total power constraint in each cell and interference constraints of users assigned to subcarrier are satisfied.

\section{B. Cognitive Two Cells Iterative Spectrum Balancing} (CTC-ISB)

In this section, we propose a solution for (3) based on the results obtained in [25]-[27]. The purpose is to provide a solution for the problem (3) in the dual domain. Assume that there are two interfering cells thus it is aimed to perform joint scheduling and power allocation for users located in the cells. Via utilizing (3), the Lagrange and the Lagrange dual function are obtained, then by differentiating the Lagrange function relative to $\mathrm{p}_{\mathrm{k}, \mathrm{n}}^{\mathrm{A}}$ and $\mathrm{p}_{1, \mathrm{n}}^{\mathrm{B}}$ we obtain the following results.

$$
\begin{aligned}
& p_{k, n \mid l}^{A^{*}}=\min \left\{\left(\frac{1}{\operatorname{Ln}(2) \lambda^{A}}-\frac{N_{0}+I_{P}+\bar{g}_{k, n}^{A} p_{l, n}^{B}}{g_{k, n}^{A}}\right)^{+}, \bar{I}_{n}^{A}\right\} \\
& p_{l, n \mid k}^{B^{*}}=\min \left\{\left(\frac{1}{\operatorname{Ln}(2) \lambda^{B}}-\frac{N_{0}+I_{P}+\bar{g}_{l, n}^{B} p_{k, n}^{A}}{g_{l, n}^{B}}\right), \bar{I}_{n}^{B}\right\}
\end{aligned}
$$

which $\mathrm{x}^{+}=\max (0, \mathrm{x})$. Since the powers $\mathrm{p}_{\mathrm{k}, \mathrm{n}}^{\mathrm{A}^{*}}$ and $\mathrm{p}_{1, \mathrm{n}}^{\mathrm{B}^{*}}$ must be optimal for the user selection $\mathrm{k}^{*}$ and $\mathrm{l}^{*}$, it is obvious that the powers $\mathrm{p}_{\mathrm{k}, \mathrm{n}}^{\mathrm{A}^{*}}$ and $\mathrm{p}_{1, \mathrm{n}}^{\mathrm{B}^{*}}$ must satisfy the necessary KarushKuhn-Tucker (KKT) optimality conditions.

To solve (3), notice that for any given feasible power allocation, the solution to

$$
\max _{k_{n}^{A}, l_{n}^{B}} C\left(P_{k, n}^{A}, P_{l, n}^{B}\right)
$$

is achieved at

$$
\begin{aligned}
& k_{n}^{A}, l_{n}^{B}=\underset{\substack{k \in K^{A} \\
l \in K^{B}}}{\arg \max _{2}}\left\{\log _{2}\left(1+\frac{g_{k, n}^{A} \cdot p_{k, n}^{A}}{N_{0}+I_{P}+\bar{g}_{k, n}^{A} p_{l, n}^{B}}\right)\right. \\
& +\log _{2}\left(1+\frac{g_{l, n}^{B} \cdot p_{l, n}^{B}}{N_{0}+I_{P}+\bar{g}_{l, n}^{B} p_{k, n}^{A}}\right) \\
& \left.-\lambda^{A} \cdot p_{k, n}^{A}-\lambda^{B} \cdot p_{l, n}^{B}-\Omega_{k, n}^{A} \cdot p_{k, n}^{A}-\Omega_{l, n}^{B} \cdot p_{l, n}^{B}\right\}
\end{aligned}
$$

On the other hand, for any given user selection $\mathrm{k} \in \mathrm{K}^{\mathrm{A}}$ and $1 \in \mathrm{K}^{\mathrm{B}}$ the corresponding optimal set of powers must satisfy the Karush-Kuhn-Tucker (KKT) conditions [23], [24]. Thus, the powers are allocated to the users that maximize (4).
The aim of dual optimization is to calculate the values of $\lambda^{\mathrm{A}}$ and $\lambda^{\mathrm{B}}$ in a way that the best bound could be calculated as shown in the following.

$$
\lambda^{A}, \lambda^{B}=\min _{\lambda^{A}, \lambda^{B}}\left\{g\left(\lambda^{A}, \lambda^{B}\right)\right\}
$$

where $g(\lambda)$ is the Lagrange dual function.

By considering that, the resource allocation in one cell depends on the resource allocation in adjacent cells, so $\lambda^{\mathrm{A}}$ and $\lambda^{\mathrm{B}}$ should be updated jointly. One of the best choices for updating dual multivariable problem is ellipsoid method. Since $g(\lambda)$ is a convex function, the mentioned dual problem can be solved by using the ellipsoid method. It is necessary to point out that even $g(\lambda)$ is convex, however it may not be differentiable and does not have gradient. Thus, a search method based on sub-gradient approach [27] is performed as follows.

$$
\begin{aligned}
& d\left(\lambda^{A}(t)\right)=P_{T}^{A}-\sum_{k=1}^{K^{A}} \sum_{n=1}^{N} p_{k, n}^{A} \\
& d\left(\lambda^{B}(t)\right)=P_{T}^{B}-\sum_{l=1}^{L^{B}} \sum_{n=1}^{N} p_{l, n}^{B}
\end{aligned}
$$

where $\left(\lambda^{A}(t), \lambda^{B}(t)\right)$ denotes the dual variables at iteration $t$. The $p_{k, n}^{A}$ and $p_{l, n}^{B}$ in the given sub-gradient at iteration $t$ can all be obtained by (11) and (12) with a specific $\left(\lambda^{A}(t), \lambda^{B}(t)\right)$. The details of the ellipsoid method, e.g., the updating of ellipsoid and dual vector, the stopping criterion, etc., can all be founded in [28].

Algorithm 2:Associated Ellipsoid Search Cognitive TwoCell Iterative Spectrum Balancing (CTC-ISB) Power Allocation

1: Initialize $\lambda^{A}(0), \lambda^{B}(0)$, and an initial ellipsoid $A(0)$;

2: repeat

3: While $\sqrt{d_{i}^{T} A_{i} d_{i}}>\varepsilon$ do

4: for each sub-carrier $\mathrm{n}=1$ to $N$ do

5: $\quad$ Calculate $P_{k, n \mid l}^{A^{*}}$ and $P_{l, n \mid k}^{B^{*}}$ via (11) and (12);

6: $\quad$ Set $k_{n}^{A}, l_{n}^{B}, p_{k, n \mid l}^{A}$, and $p_{l, n \mid k}^{B}$ according to (14), (11), and (12) respectively;

7: $\quad$ For $k_{n}^{A}=k_{n}^{A^{*}}$, set $p_{k, n \mid l}^{A}=p_{k, n \mid l}^{A^{*}}$ and $k_{n}^{A}=1$;

8: $\quad$ For $l_{n}^{B}=l_{n}^{B^{*}}$, set $p_{l, n \mid k}^{B}=p_{l, n \mid k}^{B^{*}}$ and $l_{n}^{B}=1$;

9: $\quad$ end for

10: Update dual variables $\lambda$ and ellipsoid $A$ by the ellipsoid method with sub-gradient in (16);

11: Until converge to the dual optimum $\lambda^{A^{*}}$ and $\lambda^{B^{*}}$

\section{SimUlation RESUltS}

In this section we study the performance of the proposed algorithms. 


\section{A. System Description}

In the simulation, each cell has a radius $D=500 m$ and contains the same number of users $k=l=30$. The distance between users and base stations is considered as a random variable which distributed uniformly on the interval $[50, \mathrm{D}]$. The variance of noise in each subcarrier is set to $-105 \mathrm{dBm}$. Total power of each base station is $\mathrm{P}_{\mathrm{T}}=20$ watts.

The joint resource allocation problem for cell $A$ and cell $B$ is solved for a large number of random variables containing users distance, Rayleigh fading and shadowing. The average results for sum-throughput is depicted. We provide more details for conducting the numerical results in the following.

Let us define $\mathrm{d}^{\mathrm{A}}$ and $d^{B}$ as the vectors containing the distance of all users in cell $A$ and $B$ respectively. Recall that $\forall k, l, d_{k}^{A}$ and $d_{l}^{B}$ are random variables with a uniform distribution on $[50, \mathrm{D}]$. Then, free space loss is modeled by shadowing effect $L_{k}^{A}\left(d_{k}^{A}\right)=20 \log _{10}\left(d_{k}^{A}\right)+40.04+l_{k}^{A} \quad$ and $L_{l}^{B}\left(d_{l}^{B}\right)=20 \log _{10}\left(d_{l}^{B}\right)+40.04+l_{l}^{B}$ that $1_{\mathrm{k}}^{\mathrm{A}}$ and $\mathrm{l}_{1}^{\mathrm{B}}$ is a real Gaussian random variable with zero mean and standard deviation 7 accounting for large-scale Log-normal shadowing. Due to small scale fading $H_{k, n}^{A}=10^{-\frac{L_{d B}^{A}}{20}} \times g_{k, n}^{A}$ which $g_{k, n}^{A}$ is a complex Gaussian random variable with zero mean and variance 1 accounting for Rayleigh fast fading. In the similar manner $H_{l, n}^{B}, \quad \bar{H}_{k, n}^{A}$, and $\overline{\mathrm{H}}_{1, \mathrm{n}}^{\mathrm{B}}$ are obtained. $\mathrm{I}_{\mathrm{P}}$ is interference caused by base station of PRN and is set to $-50 \mathrm{dBm} . \overline{\mathrm{I}}_{\mathrm{n}}^{\mathrm{A}}$ and $\overline{\mathrm{I}}_{\mathrm{n}}^{\mathrm{B}}$ Interference thresholds on the PRN are considered as a random variables which distributed uniformly on the interval $\left[\frac{\mathrm{P}_{\mathrm{T}}}{2 \mathrm{~N}} \frac{3 \mathrm{P}_{\mathrm{T}}}{2 \mathrm{~N}}\right]$. For each realization of $d_{k}^{A}, l_{k}^{A}$, $g_{k, n}^{A}$ and $d_{l}^{B}, l_{l}^{B}, g_{l, n}^{B}$ sum-throughput of two cells based on power allocation and subcarrier scheduling is performed.

\section{B. Numerical Results}

For all algorithms, we consider $N=64$ and $N=256$ subcarriers with interference threshold $T=0.01$ and $T=1$ on the primary network. In addition, for comparison two different algorithms are considered: 1) equal power allocation algorithm; and 2) single cell power allocation algorithm. In Fig. 2, the sum-throughput versus the number of users is depicted. Four network configurations are considered corresponding to $N=64, N=256$ and interference constraint on primary network with $T=0.01, T=1$. As shown in Fig. 2, convergence is always observed for all algorithms. For the CTC-ISB algorithm, the results in Fig. 2 confirm that the value of the objective function is monotonically increased by increasing the number of users and confirm the effect of the multiuser diversity. It is observed that the performance of proposed algorithms is better than the equal power allocation and single cell power allocation algorithm. Furthermore, by increasing interference threshold on primary network from 0.01 to 1 , the performance improvement occurs in $2 d B$ and sum-throughput of system increases from $16 d B$ to $18 d B$. Indeed, when interference threshold on PRN increases, the performance of CRN to efficiently utilize the spectrum of
PRN is maximized thus, the sum-throughput of CRN is improved in $2 d B$.

\section{CONCLUDING REMARKS}

In this paper, a number of methods have been proposed for resource allocation in cellular cognitive radio networks based on OFDMA technique, where for each user, service is provided via a base station. Two iterative algorithms were introduced and investigated, namely: 1) CSC-MAC, 2) CTCISB. In all of these approaches, each user announces the available channel share in each subcarrier and central control unit collects data and performs required processes on them.

To find the search area faster, the CSC-MAC algorithm was proposed. In this scheme, primary values for the dual variables in each cell are obtained. By calculating these amounts, the search interval becomes more restricted and as a result, the algorithm will converge to the optimal values faster. After that, we proposed a second algorithm CTC-ISB in which the purpose is sum-throughput maximization using dual optimization method. In this method, waterfilling algorithm is performed for users in each cell according to the effects caused by the users in adjacent cell, and power allocation and subcarrier scheduling are performed. Then, the dual variables are updated using ellipsoid method, and this trend is repeated till the algorithm converges to the optimal values. Simulation results have demonstrated that the performance of the proposed algorithms outperform two above mentioned algorithms as well. Furthermore, the convergence is observed in the proposed algorithms.

\section{ACKNOWLEDGMENT}

This research was supported by Research institute for ICT under Grant 12301/500.

\section{REFERENCES}

[1] Federal Communications Commission, "Spectrum policy task force report," Rep. ET Docket no. 02-135, Nov. 2002.

[2] J. I. Mitola, "Cognitive radio: making software radios more personl," IEEE Personal Commun., vol. 6, pp. 13-18, Aug. 1999. https://doi.org/10.1109/98.788210

[3] S. Haykin, "Cognitive radio: brain-empowered wireless communications," IEEE J. Sel. Areas Commun., vol. 23, pp. 201-220, Feb. 2005. https://doi.org/10.1109/JSAC.2004.839380

[4] M. Gastpar,"On capacity under receive and spatial spectrum-sharing constraints," IEEE Trans. Inform. Theory, vol. 53, no. 2, pp. 471-487, Feb 2007. https://doi.org/10.1109/TIT.2006.889016

[5] N. Devroye, P. Mitranand, and V. Tarokh, "Achievable rates in cognitive radio channels," IEEE Trans. Inform. Theory, vol. 52, no. 5, pp. 1813-1827, May 2006. https://doi.org/10.1109/TIT.2006.872971

[6] A. Jovicic and P. Viswanath, "Cognitive radio: an information-theoretic perspective, " IEEE Trans. Inform. Theory, vol. 52, no. 7, pp. 24132417, July 2006. https://doi.org/10.1109/isit.2006.262021

[7] N. Devroye, P. Mitran, and V. Tarokh, "Cognitive multiple access networks," in Proc. International Symp. Inform. Theory, Adelaide, Australia, Sept. 2005. https://doi.org/10.1109/isit.2005.1523292

[8] J. Y. Won, S. B. Shim, Y. H. Kim, S. H. Hwang, M. S. Song, and C. J. Kim, "An adaptive OFDMA platform for IEEE 802.22 based on cognitive radio," in Proc. Asia-Pacific Conf. Commun., Aug. 2006, pp. $1-5$.

https://doi.org/10.1109/apcc.2006.255809 
[9] A. Batra, S. Lingam, and J. Balakrishnan, "Multi-band OFDM: a cognitive radio for UWB," in Proc. IEEE International Symposium Circuits Syst. (ISCAS), May 2006, pp. 4094-4097. https://doi.org/10.1109/iscas.2006.1693529

[10] T. Feng and Y. Zhen, "A new algorithm for weighted proportional fairness based spectrum allocation of cognitive radios," in Proc. International Conf. Software Engineering, Artificial Intelligence, Networking, Parallel/Distributed Comput. (SNPD), July-Aug. 2007, pp. 531-536. https://doi.org/10.1109/snpd.2007.134

[11] K. Hamdi, W. Zhang, and K. Letaief, "Uplink scheduling with QoS provisioning for cognitive radio systems," in Proc. IEEE Wireless Commun. Networking Conf. (WCNC), Mar. 2007, pp. 2592-2596. https://doi.org/10.1109/wcnc.2007.482

[12] J.-A. Bazerque and G. B. Giannakis, "Distributed scheduling and resource allocation for cognitive OFDMA radios," in Proc. CrownCom, Aug. 2007, pp. 343-350.

[13] J.-A. Attar, O. Holland, M. Nakhai, and A. Aghvami, "Interferencelimited resource allocation for cognitive radio in orthogonal frequencydivision multiplexing networks," IET Commun., vol. 2, pp. 806-814, July 2008.

https://doi.org/10.1049/iet-com:20070355

[14] P. Cheng, Z. Zhang, H.-H. Chen, and P. Qiu, "Optimal distributed joint frequency, rate and power allocation in cognitive OFDMA systems," IET Commun., vol. 2, pp. 815-626, July 2008. https://doi.org/10.1049/iet-com:20070358

[15] S. Aslam, A. Shahid, and K.-G. Lee, "IMS: interference minimization scheme for cognitive radio networks using Hungarian algorithm," in Proc. IEEE Int. Conf. Future Generation Commun. Technol. (FGCT), London, UK, Dec. 2012, pp. 17-21. https://doi.org/10.1109/fgct.2012.6476564

[16] X. Tao, Z. F. Zhao, R. P. Li, J. Palicot, and H. G. Zhang, "Downlink interference minimization in cooperative cognitive LTE-femtocell networks,” EURASIP J. Wireless Commun. Netw., 2013 https://doi.org/10.1186/1687-1499-2013-194.

[17] T. Q. Duong, V. N. Q. Bao, H. Tran, G. C. Alexandropoulos, and H.-J. Zepernick, "Effect of primary network on performance of spectrum sharing AF relaying," Electron. Lett., vol. 48, no. 1, pp. 25-27, Jan. 2012. https://doi.org/10.1049/el.2011.3151

[18] Q. Wu, Z. Zhang, and J. Wang, "Outage analysis of cognitive relay networks with relay selection under imperfect CSI environment," IEEE Commu. Lett., vol. 17, no. 7, pp. 1297-1300, Jul. 2013. https://doi.org/10.1109/LCOMM.2013.052413.130424

[19] P. L. Yeoh, M. Elkashlan, T. Q. Duong, N. Yang, and D. B. da Costa, "Transmit antenna selection for interference management in cognitive relay networks," IEEE Trans. Veh. Technol., vol. 63, no. 7, pp. 32503262, Sep. 2014. https://doi.org/10.1109/TVT.2014.2298387

[20] Y. Huang, F. Al-Qahtani, Q. Wu, C. Zhong, J. Wang, and H. Alnuweiri, "Outage analysis of spectrum sharing relay systems with multiple destinations under primary user's interference," IEEE Trans. Veh. Technol., vol. 63, no. 7, pp. 3456-3464, Sep. 2014 https://doi.org/10.1109/TVT.2014.2297973

[21] V. N. Q. Bao, T. Q. Duong, and C. Tellambura, "On the performance of cognitive underlay multihop networks with imperfect channel state information," IEEE Trans. Commun., vol. 61, no. 12, pp. 4864-4873, Dec. 2013. https://doi.org/10.1109/TCOMM.2013.110413.130167

[22] J. Hussein, S. Ikki, S. Boussakta, and C. Tsimenidis, "Performance study of the dual-hop underlay cognitive network in the presence of cochannel interference," in Proc. IEEE 81st Veh. Technol. Conf. (VTC Spring), May 2015, pp. 1-5. https://doi.org/10.1109/vtcspring.2015.7146013

[23] S. Boyd and L. Vanderberghe, Convex Optimization. Cambridge, U.K: Cambridge Univ. Press, 2004 https://doi.org/10.1017/CBO9780511804441

[24] Z.-Q. Luo and W. Yu, "An introduction to convex optimization for communication and signal processing," IEEE J. Sel. Areas Commun., vol. 24, no. 8, pp. 1426-1438, Aug. 2006. https://doi.org/10.1109/JSAC.2006.879347

[25] W. Yu, R. Lui, and R. Cendrillos, "Dual optimization methods for multiuser orthogonal frequency division multiplex systems," in Proc IEEE Global Telecommun. Conf., Dallas, TX, Nov. 2004, pp. 225-229.
[26] R. Cendrillon, W. Yu, M. Moonen, J. Verlinden, and T. Bostoen, "Optimal multiuser spectrum balancing for digital subscriber lines," IEEE Trans. Commun, vol. 54, no. 5, pp. 922-933, May 2006. https://doi.org/10.1109/TCOMM.2006.873096

[27] W. Yu and R. Lui, "Dual methods for nonconvex spectrum optimization of multicarrier systems," IEEE Trans. Commun., vol. 54, no. 7, pp. 1310-1322, Jul. 2006. https://doi.org/10.1109/TCOMM.2006.877962

[28] Lecture Slides on Class EE364b of Stanford Univ., S. Boyd, Ellipsoid Method, 2015.

[Online]. Available:http://www.stanford.edu/class/ee364b/lectures.html

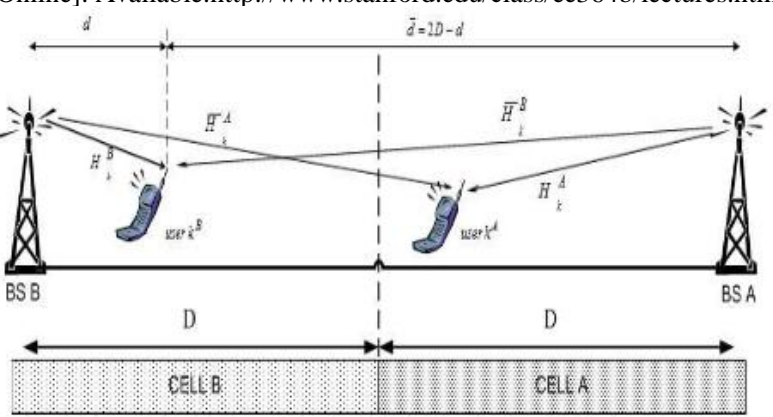

Figure 1: Two cell cognitive radio system model

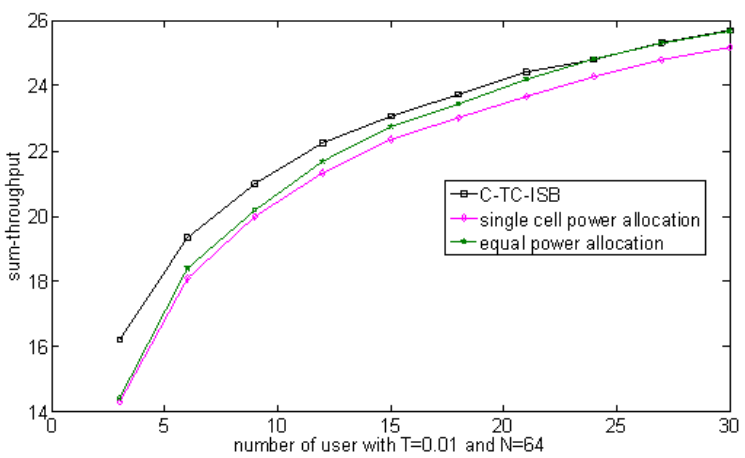

(a)

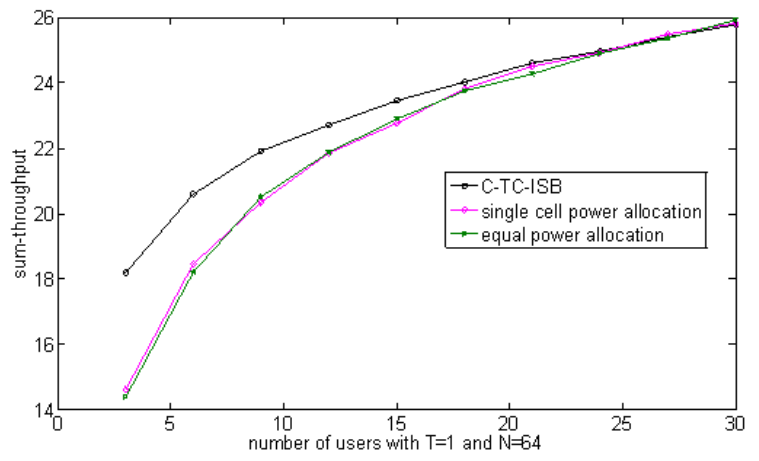

(b)

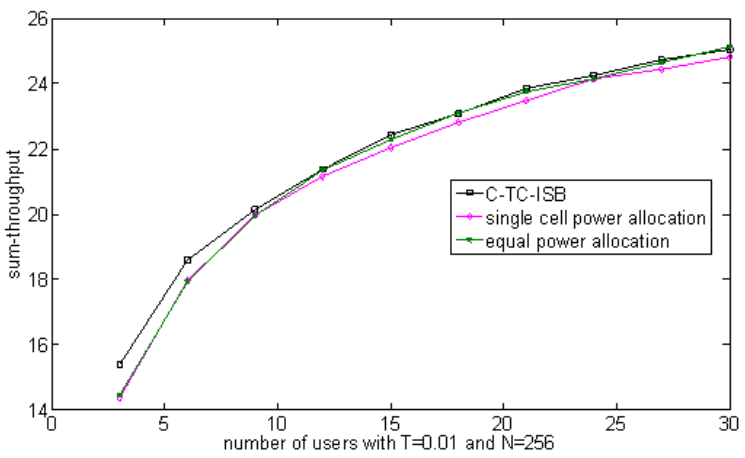


(c)

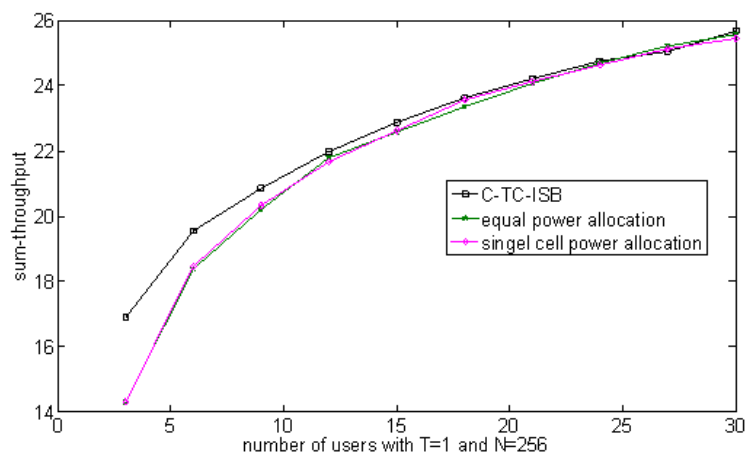

(d)

Figure 2: Sum-throughput versus the number of users: CTC-ISB, with $\mathrm{N}=64$ and $\mathrm{N}=256$ sub-carriers and interference threshold on $\mathrm{PRN}$ with $\mathrm{T}=0.01$ and $\mathrm{T}=1$. The performance of the proposed algorithms is compared with equal power allocation algorithm and single cell power allocation. As shown in the figure, the proposed algorithms outperform the aforementioned algorithms. 